\title{
Article \\ Diagnostic Performance of the Fujifilm SILVAMP TB-LAM in Children with Presumptive Tuberculosis
}

\author{
Patricia Comella-del-Barrio ${ }^{1}{ }^{\circledR}$, Bárbara Molina-Moya ${ }^{1}$, Jacqueline Gautier ${ }^{2}$, Raquel Villar-Hernández ${ }^{1}{ }^{\circledR}$, \\ Mariette Jean Coute Doresca ${ }^{2}$, Beatriz Sallés-Mingels ${ }^{3}$, Lydia Canales-Aliaga ${ }^{4}$, Margareth Narcisse ${ }^{2}$, \\ Tomás M. Pérez-Porcuna ${ }^{4}\left(\mathbb{D}\right.$, Jacob Creswell ${ }^{5} \mathbb{C}$, Luis E. Cuevas ${ }^{6,+}+\mathbb{D}$ and José Domínguez ${ }^{1, *,+}$
}

Citation: Comella-del-Barrio, P.; Molina-Moya, B.; Gautier, J.; Villar-Hernández, R.; Doresca, M.J.C.; Sallés-Mingels, B.; Canales-Aliaga, L.; Narcisse, M.; Pérez-Porcuna, T.M.;

Creswell, J.; et al. Diagnostic

Performance of the Fujifilm SILVAMP TB-LAM in Children with Presumptive Tuberculosis. J. Clin. Med. 2021, 10, 1914. https://doi.org/ 10.3390/jcm10091914

Academic Editor: Luis Garcia-Marcos

Received: 24 March 2021

Accepted: 23 April 2021

Published: 28 April 2021

Publisher's Note: MDPI stays neutral with regard to jurisdictional claims in published maps and institutional affiliations.

Copyright: (c) 2021 by the authors. Licensee MDPI, Basel, Switzerland. This article is an open access article distributed under the terms and conditions of the Creative Commons Attribution (CC BY) license (https:// creativecommons.org/licenses/by/ $4.0 /)$.
1 Institut d'Investigació Germans Trias i Pujol, CIBER Enfermedades Respiratorias, Universitat Autònoma de Barcelona, 08916 Barcelona, Spain; patricia.comella@e-campus.uab.cat (P.C.-d.-B.); bmolina@igtp.cat (B.M.-M.); raquel.villarhernandez@gmail.com (R.V.-H.)

2 Department of Pediatrics, Division of Tuberculosis, Hôpital Saint-Damien, Nos Petits-Frères et Sœurs, Port-au-Prince 6112, Haiti; jacqueline.gautier@nph.org (J.G.); mamaille71@yahoo.fr (M.J.C.D.); margareth.narcisse@nph.org (M.N.)

3 Department of Radiology and Imaging Diagnose, Centre d'Atenció Primària (CAP) Manso, 08015 Barcelona, Spain; reyes.salles@gmail.com

4 Servei de Pediatria, Atenció Primària, Unitat de Investigació Fundació Mútua Terrassa, Hospital Universitari Mútua Terrassa, 08221 Barcelona, Spain; lcanales@mutuaterrassa.es (L.C.-A.); tomas.perez.porcuna@gmail.com (T.M.P.-P.)

5 Stop TB Partnership, TB Reach, 1218 Geneva, Switzerland; jacobc@stoptb.org

6 Department of Clinical Sciences, Liverpool School of Tropical Medicine, Liverpool L3 5QA, UK; Luis.Cuevas@1stmed.ac.uk

* Correspondence: jadominguez@igtp.cat; Tel.: +34-93-033-0537

+ Senior co-authors.

Abstract: Current diagnostics for tuberculosis (TB) only manage to confirm a small proportion of children with TB and require respiratory samples, which are difficult to obtain. There is a need for non-invasive biomarker-based tests as an alternative to sputum testing. Fujifilm SILVAMP TB lipoarabinomannan (FujiLAM), a lateral-flow test to detect lipoarabinomannan in urine, is a novel non-sputum-based point-of-care diagnostic reported to have increased sensitivity for the diagnosis of TB among human immunodeficiency virus (HIV)-infected adults. We evaluate the performance of FujiLAM in children with presumptive TB. Fifty-nine children attending a paediatric hospital in Haiti with compatible signs and symptoms of TB were examined using Xpert MTB/RIF, smear microscopy and X-rays, and classified according to the certainty of diagnosis into bacteriologically confirmed TB $(n=5)$, unconfirmed TB (bacteriologically negative, $n=50)$ and unlikely TB $(n=4)$. Healthy children $(n=20)$ were enrolled as controls. FujiLAM sensitivity and specificity were $60 \%$ and $95 \%$ among children with confirmed TB. FujiLAM's high specificity and its characteristics as a point-of-care indicate the test has a good potential for the diagnosis of TB in children.

Keywords: tuberculosis; diagnosis; lipoarabinomannan; LAM; children; urine; point-of-care

\section{Introduction}

Tuberculosis (TB) is a major cause of morbidity and mortality in children [1]. However, the paucibacillary nature of TB in children and the difficulty of obtaining respiratory samples create significant barriers for diagnosis [2]. Most children with TB, especially in countries with high TB burden [3], are not bacteriologically confirmed, or are missed [3]. The lack of reliable diagnostic methods has highlighted the need for non-sputum-based tests for childhood TB [4,5].

In recent years, attempts have been made to develop diagnostic tests based on the detection of lipoarabinomannan (LAM) antigen, a lipoglycan and virulence factor in the bacteria genus Mycobacterium present in the outer cell wall, which is released from 
metabolically active or degenerating bacterial cells. As LAM is heat stable, filtered by the kidney and detectable in the urine of people with overt TB, test prototypes have attempted to identify LAM in urine since the 1930s [6,7]. Until recently, urine-based LAM prototypes have had low sensitivity and performed better in individuals co-infected with human immunodeficiency virus (HIV) and advanced immunosuppression [8]. Although the biological mechanisms for the better performance in HIV-positive individuals are not fully understood, the higher concentration of LAM in the urine could be due to a greater difficulty in containing Mycobacterium tuberculosis with subsequent hematogenous spread to the kidneys or to a higher body burden of M. tuberculosis [9-12]. Compared to the Alere Determine TB-LAM assay, with a reported sensitivity of about $40 \%$ for detecting TB in HIV-infected patients [13], a new assay is reported to have higher sensitivity to detect LAM in both HIV-infected and uninfected patients [14,15]. The assay, the Fujifilm SILVAMP TB LAM (FujiLAM, Fujifilm, Tokyo, Japan), combines high-affinity monoclonal antibodies against $M$. tuberculosis-specific LAM epitopes and a silver amplification step to increase the visibility of the test and control lines [15]. However, data on its performance in children is limited, and we report here its performance in a case series of children with signs and symptoms of presumptive TB. TB in children has more disseminated clinical presentations than adults because of their poor containment of TB. In addition, children have difficulty in expectorating sputum, and assays that use non-sputum samples could be useful, particularly in young children $[2,11,16-18]$.

\section{Materials and Methods}

This was a retrospective case series of the bio-banked urine samples of 59 children with signs and symptoms compatible with $\mathrm{TB}$, and of 20 healthy control children. Children were enrolled prospectively between August 2015 and December 2016 [19]. The children with presumptive TB were between 0 and 14 years old, and were enrolled when attending the Saint Damien paediatric reference hospital in Port-au-Prince, Haiti. Children were initially identified by the diagnostic clinics and enrolled opportunistically. This is a case series, as we did not estimate the number of children required and instead enrolled as many children being investigated for $\mathrm{TB}$ as possible over a period of sixteen months. The healthy children, enrolled as controls, were attending a primary school in the same neighbourhood of the hospital and were between 5 and 8 years old. The objective of including a control group was to explore whether any of the children without symptoms of TB had a positive FujiLAM test to better characterize the specificity of the test.

After obtaining informed parental consent, we collected clinical and demographic data, vaccination history (including Bacillus Calmette-Guérin (BCG)) and information about current and previous medications. Children were excluded if they had known immunodeficiencies, were receiving immunosuppressive treatment, or if they had received anti-TB treatment for two or more weeks before enrolment. Tuberculin skin tests (TSTs) were performed by a trained technician using $0.1 \mathrm{~mL}$ of Tubersol (bioequivalent to 5 Tuberculin Units; Sanofi Pasteur, Canada) and were considered TST positive if they had $\geq 10 \mathrm{~mm}$ induration in the presence of a BCG scar or $\geq 5 \mathrm{~mm}$ in non-BCG-vaccinated children with no known contact with adults with TB [20]. Three millilitres of blood was obtained for the QuantiFERON-TB Gold In-Tube test (QFT-GIT, Qiagen, Germany [21]), following the manufacturer's instructions.

Children with presumptive TB underwent anterior-posterior chest X-rays, which were read by two radiologists blinded to the child's condition, and a third reader resolved reading disagreements. Three consecutive sputum samples obtained by induced or nasopharyngeal/nasogastric aspiration were examined by fluorescence smear microscopy stained with auramine, and children with positive smear microscopy or abnormal X-rays underwent Xpert MTB/RIF (Cepheid, Sunnydale, CA, USA) following local diagnostic algorithms. No culture facilities were available. Children with lymph node adenopathy underwent biopsies for histological examination. 
Children with presumptive TB were classified using the clinical case definitions into confirmed, unconfirmed, and unlikely TB [22]. Children were classified as having confirmed TB if bacteriologically confirmed by Xpert MTB/RIF; as unconfirmed TB if there was no bacteriological confirmation but a positive TST or QFT-GIT and at least one of the clinical criteria of the Clinical Case Definitions (i.e., X-rays consistent with TB, signs and symptoms of $\mathrm{TB}$, close $\mathrm{TB}$ exposure, or positive response to $\mathrm{TB}$ treatment), or at least two clinical criteria if the TST and QFT-GIT results were negative; and unlikely TB if the child had only evidence of $M$. tuberculosis infection or presented only one clinical criterion compatible with TB. Enrolled school children had a negative TST and QFT-GIT, and no signs or symptoms of TB. Those with positive TST or symptoms compatible with TB were referred to the hospital for TB screening and were not included as controls.

Participants were asked to provide a midstream urine sample on-site, collected in sterile plastic containers, or in urine bags for children $<1$ year, and stored at room temperature if they were processed at the time of collection, or stored in a refrigerator if processed in batches at the end of the day. Urine samples were aliquoted $(2 \mathrm{~mL})$ into cryovials and kept at $-20{ }^{\circ} \mathrm{C}$ until processing in Badalona, Spain (IGTP). To test with FujiLAM, urine samples were thawed at room temperature, mixed with a vortex, and processed following the manufacturer's instructions steps: first, the reagent tube was filled with urine up to the indicator line, mixed and left to incubate for $40 \mathrm{~min}$ at room temperature. The tube was then mixed, two drops of urine were added to the test device, and the first button was pressed. After 3-10 min of incubation, the second button was pressed to release the silver ions around the gold-conjugated antibody. The results were then read after one minute by two investigators blinded to the participants' TB status, following the manufacturer's instructions. If readers disagreed on the presence of test lines, the test was repeated once.

Categorical variables were described by frequencies and percentages, and quantitative variables described using means and $95 \%$ confidence intervals (CIs) if normally distributed, and medians and interquartile ranges (IQRs) if not normally distributed. We used Fisher's exact test, chi-square and Kruskal-Wallis tests to compare between groups, and $p$-values $<0.05$ were considered statistically significant. The sensitivity, specificity, and predictive values of FujiLAM were estimated using Xpert MTB/RIF as the microbiological reference standard, and against a composite of the combination of clinical diagnosis and Xpert MTB/RIF. Diagnostic accuracy was calculated for microbiologically confirmed cases vs. cases with unconfirmed $\mathrm{TB}$, unlikely $\mathrm{TB}$ or controls, and for the composite reference standard vs. unlikely TB and controls. Statistical analysis was performed using SPSS (SPSS version 26.0, SPSS Inc, Chicago, IL, USA). The World Health Organization (WHO) Anthro 3.2.2 ( $<5$ years) and Anthro Plus 1.0.4 ( $\geq 5$ years) were used to estimate $z$-scores $[23,24]$. Undernutrition was classified as a weight-for-age $z$-score $<-2$ (or as body mass index-for-age $z$-score $<-2$ for children above 10 years) and stunting as a height-for-age $z$-score $<-2$.

Informed consent was obtained from the parents or legal guardians. The study was approved by the Ethics Committee of the University of Barcelona and the Haiti National Ethics Committee (reference number IRB00003099).

\section{Results}

Eighty-two children were enrolled, of which three were excluded due to insufficient urine volumes. Of the 59 with presumptive TB, 5 were bacteriologically confirmed, 50 had unconfirmed but clinically diagnosed TB, and 4 were deemed not to have TB. The additional 20 were healthy controls. Demographic characteristics of the study groups are shown in Table 1. Fifty-one (64.6\%) children were male, with a median age of 76 (IQR $58-121)$ months. Their median body mass index was 14.80 (SD \pm 2.4 ); eighteen (30.5\%) reported recent and severe weight loss and twelve (20.7\%) reported stunted growth. Fiftysix $(75.7 \%)$ had received the BCG vaccine and had a BCG scar. FujiLAM results are shown in Table 2. Eight (10.1\%) samples were positive and seventy-one ( $89.9 \%$ ) negative (Table 2). Three of the positive samples belonged to children with bacteriologically confirmed $\mathrm{TB}$, three to children with unconfirmed TB, one to a child with unlikely TB and one to a 
control. Among the six FujiLAM-positive children with bacteriologically confirmed and unconfirmed $\mathrm{TB}$, four had intrathoracic and one both intrathoracic and TB spondylitis. If Xpert MTB/RIF was considered the reference standard, the sensitivity of FujiLAM was $60 \%$ (95\% CI 17-93) and the specificity, considering the control group, 95\% (95\% CI 73-100). If confirmed and unconfirmed TB were compared against unlikely TB and controls, the sensitivity was $11 \%$ (95\%CI 5-23) and the specificity $92 \%$ (95\% CI $72-99)$. Five (71.4\%) of the seven FujiLAM-positive children with presumptive TB were underweight and four $(66.7 \%)$ were stunted ( $p=0.023$ and $p=0.014$, respectively). The one case of disagreement of FujiLAM assays between the two readers was resolved after repeating the test.

Table 1. Demographic and clinical characteristics of participants.

\begin{tabular}{|c|c|c|c|c|c|c|}
\hline & Overall $(n=79)$ & $\begin{array}{l}\text { Confirmed TB } \\
\qquad(n=5)\end{array}$ & $\begin{array}{l}\text { Unconfirmed } \\
\text { TB }(n=50)\end{array}$ & $\begin{array}{l}\text { Unlikely TB } \\
\qquad(n=4)\end{array}$ & $\begin{array}{l}\text { Controls } \\
(n=20)\end{array}$ & $\begin{array}{c}p- \\
\text { Value }\end{array}$ \\
\hline Female & $28(35 \%)$ & $1(20 \%)$ & $21(42 \%)$ & $1(25 \%)$ & $5(25 \%)$ & 0.530 \\
\hline Male & $51(65 \%)$ & $4(80 \%)$ & $29(58 \%)$ & $3(75 \%)$ & $15(75 \%)$ & \\
\hline Median age (IQR) months & $76(58-121)$ & $95(51-128)$ & $76(51-122)$ & $152(98-165)$ & $70(58-94)$ & 0.109 \\
\hline$<5$ yrs & $24(30 \%)$ & $1(20 \%)$ & $16(32 \%)$ & $0(0 \%)$ & $7(35 \%)$ & 0.654 \\
\hline$\geq 5$ yrs. & $55(70 \%)$ & $4(80 \%)$ & $34(68 \%)$ & $4(100 \%)$ & $13(65 \%)$ & \\
\hline BCG scar $(n=74)$ & $56(76 \%)$ & $3(60 \%)$ & $35(78 \%)$ & $2(50 \%)$ & $16(80 \%)$ & 0.390 \\
\hline TST or QFT-GIT positive & $55(93 \%)$ & $5(100 \%)$ & $46(92 \%)$ & $4(100 \%)$ & $0(0 \%)$ & 1.000 \\
\hline TST positive & $52(88 \%)$ & $4(80 \%)$ & $44(88 \%)$ & $4(100 \%)$ & $0(0 \%)$ & 0.707 \\
\hline QFT-GIT positive $(n=53)$ & $37(70 \%)$ & $5(100 \%)$ & $28(64 \%)$ & $4(100 \%)$ & $0(0 \%)$ & 0.101 \\
\hline TB contact & $50(85 \%)$ & $1(20 \%)$ & $45(90 \%)$ & $4(100 \%)$ & $0(0 \%)$ & 0.002 \\
\hline Cough & $48(81 \%)$ & $5(100 \%)$ & $39(78 \%)$ & $4(100 \%)$ & $0(0 \%)$ & 0.491 \\
\hline Fever & $40(68 \%)$ & $4(80 \%)$ & $34(68 \%)$ & $2(50 \%)$ & $0(0 \%)$ & 0.718 \\
\hline Lethargy & $4(7 \%)$ & $3(60 \%)$ & $1(2 \%)$ & $0(0 \%)$ & $0(0 \%)$ & 0.002 \\
\hline Weight loss $(n=56)$ & $19(34 \%)$ & $3(60 \%)$ & $15(32 \%)$ & $1(25 \%)$ & $0(0 \%)$ & 0.517 \\
\hline Adenopathy & $22(37 \%)$ & $4(80 \%)$ & $18(36 \%)$ & $0(0 \%)$ & $0(0 \%)$ & 0.046 \\
\hline Underweight $(n=74)$ & $18(31 \%)$ & $3(60 \%)$ & $15(30 \%)$ & $0(0 \%)$ & $0(0 \%)$ & 0.201 \\
\hline Stunted $(n=58)^{\mathrm{a}}$ & $12(21 \%)$ & $2(40 \%)$ & $9(18.4 \%)$ & $1(25 \%)$ & & 0.418 \\
\hline X-ray consistent with TB & $28(46 \%)$ & $5(100 \%)$ & $23(46 \%)$ & $0(0 \%)$ & NA & 0.161 \\
\hline $\begin{array}{l}\text { Positive smear-microscopy } \\
\qquad(n=52)\end{array}$ & $10(19 \%)$ & $3(60 \%)$ & $7(16 \%)$ & $0(0 \%)$ & $0(0 \%)$ & 0.062 \\
\hline Treatment completed & $50(85 \%)$ & $5(100 \%)$ & $45(90 \%)$ & $0(0 \%)$ & NA & $<0.001$ \\
\hline Lost to follow-up & $8(14 \%)$ & $0(0 \%)$ & $4(8 \%)$ & $4(100 \%)$ & NA & \\
\hline Died & $1(2 \%)$ & $0(0 \%)$ & $1(2 \%)$ & $0(0 \%)$ & NA & \\
\hline Intrathoracic & $18(31 \%)$ & $2(40 \%)$ & $16(32 \%)$ & $0(0 \%)$ & NA & $<0.001$ \\
\hline Extra-thoracic & $7(12 \%)$ & $0(0 \%)$ & $7(14 \%)$ & $0(0 \%)$ & NA & \\
\hline Both & $4(7 \%)$ & $3(60 \%)$ & $1(2 \%)$ & $0(0 \%)$ & NA & \\
\hline Not defined & $30(51 \%)$ & $0(0 \%)$ & $26(52 \%)$ & $4(100 \%)$ & NA & \\
\hline
\end{tabular}

IQR: interquartile range; BCG: Bacillus Calmette-Guérin; TST: tuberculin skin test; QFT: QuantiFERON-TB Gold. Malnutrition defined as weight-for-age $z$-score $<-2$ for children under 10 years old and body mass index -for-age $z$-score $<-2$ for children above 10 years. Stunting was defined as height-for-age $<-2$. ${ }^{a}$ Controls have no results on stunting because height measurements were not taken.

Table 2. FujiLAM results by study group.

\begin{tabular}{cccc}
\hline & \multicolumn{3}{c}{ FujiLAM } \\
\cline { 2 - 4 } & Positive $\boldsymbol{n}(\boldsymbol{\%})$ & Negative $\boldsymbol{n} \mathbf{( \% )}$ & Total \\
\hline Confirmed TB & $3(60 \%)$ & $2(40 \%)$ & 5 \\
Unconfirmed TB & $3(6 \%)$ & $47(94 \%)$ & 50 \\
Unlikely TB & $1(25 \%)$ & $3(75 \%)$ & 4 \\
Controls & $1(5 \%)$ & $19(95 \%)$ & 20 \\
All & $8(10.1 \%)$ & $71(89.9 \%)$ & 79 \\
\hline
\end{tabular}

\section{Discussion}

We evaluated the FujiLAM test in a case series of children attending the hospital with signs and symptoms of presumptive TB. Among children with bacteriologically 
confirmed TB, the test had a sensitivity of $60 \%$, with a high specificity of $95 \%$. However, the case series is small, which resulted in wide and overlapping 95\% CI, and therefore larger studies are needed to confirm our findings. The current LAM assay (AlereLAM) recommended by the WHO in adults [8] and children $[25,26]$ is only recommended for severely immunosuppressed adults with HIV and CD4 counts $<100$ cells $/ \mu L$ [8]. Therefore, a test that performs well in HIV-uninfected patients would be a significant improvement. Unlike its predecessor, FujiLAM combines a pair of high-affinity monoclonal antibodies targeting largely $M$. tuberculosis-specific LAM epitopes and a silver amplification step to improve the visibility of the test and control lines, allowing the detection of much lower LAM concentrations in urine [6,15]. Furthermore, the FujiLAM test identified three more children who had a clinical diagnosis of TB but could not be bacteriologically confirmed, which would have lent more support to the decision to treat.

Children have a high risk of developing disseminated forms of TB, and the hematogenous and lymphatic spread of $M$. tuberculosis is likely to result in high amounts of LAM in urine [2,9]. However, few studies have reported the performance of FujiLAM in children $[27,28]$. A multicentre study in four African countries reported a relatively high sensitivity of $67.5 \%$ in HIV-negative children [28], but a cohort study in South Africa reported a lower sensitivity of 38.8\% [27]. The authors concluded that the differences in their results may have been due to the severity of the TB episodes, and that some of the studies included a high proportion of malnourished children [28]. It has been reported that LAM detection in urine is associated to the overall amount of $M$. tuberculosis in the body, as well as the severity of the disease $[10,29]$. In our study, sensitivity was higher among bacterially confirmed cases, who are likely to have more advanced disease stages, while sensitivity was lower when children with a clinical diagnosis were included. It is unlikely that all children with a clinical diagnosis of TB had TB (and thus some were misclassified), and it is thus difficult to classify a negative FujiLAM test as false negative. Furthermore, the number of positive cases was proportionally higher among underweight and stunted children [30-32]. Malnutrition has an impact in the several faces of the immune response against infections, including TB; this limits the contention capacity of the bacilli, increasing the amount of LAM in urine samples and, therefore, the sensitivity of the LAM tests [28].

FujiLAM specificity was high, which is consistent with previous findings in adults and children using microbiological (95\%) and composite reference standards (91.7\%) [10,27,28,33,34]. Although the test did not reach the $98 \%$ specificity recommended by the WHO, our sample size is not sufficiently powered to make this distinction and the $95 \%$ CI overlap with this value [35].

The FujiLAM's main advantage is its urine-based format, making an easy to do and non-invasive test without the need for additional instrumentation [10], generating opportunities to diagnose TB in patients who do not excrete bacilli, such as people with extrapulmonary or military TB, HIV and children [4]. Especially among children, the test may be able to add value to clinical decision-making as a rule in test for $\mathrm{TB}$, in our study effectively doubling the number of children treated who had confirmation of TB disease. To our knowledge, there are no studies evaluating the economic impact of LAM testing within the diagnostic algorithms for paediatric TB [36]. The inclusion of a test with the performance of the FujiLAM assay could simplify these algorithms and potentially diagnose a greater number of children with TB and increase the number of children initiating specific therapy [37]. Our study has significant limitations, including its small sample size-especially the small number of children with bacteriologically confirmed TB, which resulted in underpowered estimates. In addition, we did not culture the samples, and Xpert MTB/RIF and clinical diagnosis were used as reference standards. Xpert MTB/RIF is known to have lower sensitivity than culture [22], and thus it is likely it underestimated the number of children with bacteriologically confirmed TB. Other limitations concern retrospective testing of stored samples; however, studies using the FujiLAM test have shown good agreement between fresh and frozen urine samples [31,38]. 
In conclusion, FujiLAM had moderate sensitivity and high specificity in children compared to Xpert MTB/RIF, can process samples that are easier to collect than sputum, and can add a level of certainty to clinical diagnosis. These performance characteristics and the test's operational characteristics would facilitate a rapid diagnosis in non-sputum samples in children-especially in low- and middle-income countries, where facilities available are limited. More extensive evaluations in children are warranted.

Author Contributions: Conceptualization, L.E.C., J.D.; methodology, P.C.-d.-B., B.M.-M., J.G., R.V.-H., M.J.C.D., M.N.; validation, L.E.C., J.D.; formal analysis, P.C.-d.-B., L.E.C., J.D.; investigation, P.C.-d.-B.; resources, T.M.P.-P., J.C., J.D.; data curation, P.C.-d.-B., B.M.-M., B.S.-M.; L.C.-A., T.M.P.-P.; writingoriginal draft, P.C.-d.-B., J.D., L.E.C.; writing—review and editing, all authors; supervision, T.M.P.-P., L.E.C., J.D.; project administration, J.D.; funding acquisition, T.M.P.-P., J.C., L.E.C., J.D. All authors have read and agreed to the published version of the manuscript.

Funding: This study was supported by the Stop TB Partnership TB REACH, award from the Instituto de Salud Carlos III (DTS18/0092, FIS19/01408), integrated into the Plan Nacional de I+D+I, and cofunded by the ISCIII Subdirección General de Evaluación and the European Reginal Development Fund (ERDF); and from the European Union's Horizon 2020 Research and Innovation Programme under the Marie Skłodowska-Curie grant agreement no. 823854 (INNOVA4TB). TB REACH is supported by grant number CA-3-D000920001. https://w05.international.gc.ca/projectbrowserbanqueprojets / projectprojet/details / d000920001 (accessed on 17 March 2021).

Institutional Review Board Statement: The study was conducted according to the guidelines of the Declaration of Helsinki, and approved by the Ethics Committee of the University of Barcelona and the Haiti National Ethics Committee (reference number IRB00003099; 30 July 2015).

Informed Consent Statement: Informed consent was obtained from the parents or legal guardians of all children included in the study.

Data Availability Statement: Not applicable.

Conflicts of Interest: The authors declare no conflict of interest. The sponsors had no role in the design, execution, interpretation, or writing of the study.

\section{References}

1. Dodd, C.E.; Schlesinger, L.S. New concepts in understanding latent tuberculosis. Curr. Opin. Infect. Dis. 2017, 30, 316-321. [CrossRef] [PubMed]

2. Perez-Velez, C.M.; Roya-Pabon, C.L.; Marais, B.J. A systematic approach to diagnosing intra-thoracic tuberculosis in children. J. Infect. 2017, 74, S74-S83. [CrossRef]

3. World Health Organization. Global Tuberculosis Report 2020; WHO: Geneva, Switzerland, 2020.

4. Denkinger, C.M.; Kik, S.V.; Cirillo, D.M.; Casenghi, M.; Shinnick, T.; Weyer, K.; Gilpin, C.; Boehme, C.C.; Schito, M.; Kimerling, M.; et al. Defining the needs for next generation assays for tuberculosis. J. Infect. Dis. 2015, 211, S29-S38. [CrossRef] [PubMed]

5. Togun, T.O.; MacLean, E.; Kampmann, B.; Pai, M. Biomarkers for diagnosis of childhood tuberculosis: A systematic review. PLoS ONE 2018, 13, 1-19. [CrossRef]

6. Sigal, G.B.; Pinter, A.; Lowary, T.L.; Kawasaki, M.; Li, A.; Mathew, A.; Tsionsky, M.; Zheng, R.B.; Plisova, T.; Shen, K.; et al. A novel sensitive immunoassay targeting the 5-Methylthio-D- Xylofuranose-Lipoarabinomannan epitope meets the WHO's performance target for tuberculosis diagnosis. J. Clin. Microbiol. 2018, 56, 1-17. [CrossRef] [PubMed]

7. $\quad$ Parker, M.E. Complement fixation with urine in tuberculosis. Am. Rev. Tuberc. 1931, 23, 733-738.

8. World Health Organization. Lateral Flow Urine Lipoarabinomannan Assay (LF-LAM) for the Diagnosis of Active Tuberculosis in People Living With HIV; WHO: Geneva, Switzerland, 2019.

9. Lawn, S.D.; Gupta-Wright, A. Detection of lipoarabinomannan (LAM) in urine is indicative of disseminated TB with renal involvement in patients living with HIV and advanced immunodeficiency: Evidence and implications. Trans. R. Soc. Trop. Med. Hyg. 2015, 110, 180-185. [CrossRef] [PubMed]

10. Cox, J.A.; Lukande, R.L.; Kalungi, S.; Van Marck, E.; Van De Vijver, K.; Kambugu, A.; Nelson, A.M.; Colebunders, R.; Manabe, Y.C. Is urinary lipoarabinomannan the result of renal tuberculosis? Assessment of the renal histology in an autopsy cohort of Ugandan HIV-infected adults. PLoS ONE 2015, 10. [CrossRef] [PubMed]

11. Wood, R.; Racow, K.; Bekker, L.G.; Middelkoop, K.; Vogt, M.; Kreiswirth, B.N.; Lawn, S.D. Lipoarabinomannan in urine during tuberculosis treatment: Association with host and pathogen factors and mycobacteriuria. BMC Infect. Dis. 2012, 12, 47. [CrossRef] [PubMed] 
12. Bulterys, M.A.; Wagner, B.; Redard-Jacot, M.; Suresh, A.; Pollock, N.R.; Moreau, E.; Denkinger, C.M.; Drain, P.K.; Broger, T. Point-of-care urine LAM tests for tuberculosis diagnosis: A status update. J. Clin. Med. 2019, 9, 111. [CrossRef]

13. Bjerrum, S.; Schiller, I.; Dendukuri, N.; Kohli, M.; Nathavitharana, R.R.; Zwerling, A.A.; Denkinger, C.M.; Steingart, K.R.; Shah, M. Lateral flow urine lipoarabinomannan assay for detecting active tuberculosis in people living with HIV. Cochrane Database Syst. Rev. 2019, CD011420. [CrossRef]

14. Kerkhoff, A.D.; Sossen, B.; Schutz, C.; Reipold, E.I.; Trollip, A.; Moreau, E.; Schumacher, S.G.; Burton, R.; Ward, A.; Nicol, M.P.; et al. Diagnostic sensitivity of SILVAMP TB-LAM (FujiLAM) point-of-care urine assay for extra-pulmonary tuberculosis in people living with HIV. Eur. Respir. J. 2020, 55, 1901259. [CrossRef]

15. Broger, T.; Sossen, B.; du Toit, E.; Kerkhoff, A.D.; Schutz, C.; Ivanova Reipold, E.; Ward, A.; Barr, D.A.; Macé, A.; Trollip, A.; et al. Novel lipoarabinomannan point-of-care tuberculosis test for people with HIV: A diagnostic accuracy study. Lancet Infect. Dis. 2019, 19, 852-861. [CrossRef]

16. Rangaka, M.X.; Cavalcante, S.C.; Marais, B.J.; Thim, S.; Martinson, N.A.; Swaminathan, S.; Chaisson, R.E. Controlling the seedbeds of tuberculosis: Diagnosis and treatment of tuberculosis infection. Lancet 2015, 386, 2344-2353. [CrossRef]

17. Marais, B.J.; Schaaf, H.S. Tuberculosis in children. Cold Spring Harb. Perspect. Med. 2014, 4, 168-178. [CrossRef]

18. Marais, B.J. Childhood tuberculosis—out of the shadows. Pneumonia 2016, 8, 1-2. [CrossRef]

19. Comella-del-Barrio, P.; Abellana, R.; Villar-Hernández, R.; Coute, M.D.J.; Mingels, B.S.; Aliaga, L.C.; Narcisse, M.; Gautier, J.; Ascaso, C.; Latorre, I.; et al. A model based on the combination of IFN- $\gamma$, IP-10, ferritin and 25-Hydroxyvitamin D for discriminating latent from active tuberculosis in children. Front. Microbiol. 2019, 10, 1-15. [CrossRef]

20. Dunlap, N.E.; Bass, J.; Fujiwara, P.; Hopewell, P.; Horsburgh, C.R.; Salfinger, M.; Simone, P.M. Diagnostic standards and classification of tuberculosis in adults and children. Am. J. Respir. Crit. Care Med. 2000, 161, 1376-1395. [CrossRef]

21. Domínguez, J.; Ruiz-Manzano, J.; De Souza-Galvão, M.; Latorre, I.; Milà, C.; Blanco, S.; Jiménez, M.Á.; Prat, C.; Lacoma, A.; Altet, N.; et al. Comparison of two commercially available gamma interferon blood tests for immunodiagnosis of tuberculosis. Clin. Vaccine Immunol. 2008, 15, 168-171. [CrossRef]

22. Graham, S.M.; Cuevas, L.E.; Jean-Philippe, P.; Browning, R.; Casenghi, M.; Detjen, A.K.; Gnanashanmugam, D.; Hesseling, A.C.; Kampmann, B.; Mandalakas, A.; et al. Clinical case definitions for classification of intrathoracic tuberculosis in children: An update. Clin. Infect. Dis. 2015, 61, S179-S187. [CrossRef]

23. World Health Organization. WHO AnthroPlus for Personal Computers Manual: Software for Assessing Growth of the World's Children and Adolescents; WHO: Geneva, Switzerland, 2009.

24. World Health Organization. WHO Anthro for Personal Computers, Version 3.2.2, 2011: Software for Assessing Growth and Development of the World's Children; WHO: Geneva, Switzerland, 2010; p. 14.

25. Iskandar, A.; Nursiloningrum, E.; Arthamin, M.Z.; Olivianto, E.; Chandrakusuma, M.S. The diagnostic value of urine lipoarabinomannan (LAM) antigen in childhood tuberculosis. J. Clin. Diagn. Res. 2017, 11, EC32-EC35. [CrossRef]

26. Nicol, M.P.; Allen, V.; Workman, L.; Isaacs, W.; Munro, J.; Pienaar, S.; Black, F.; Adonis, L.; Zemanay, W.; Ghebrekristos, Y.; et al. Urine lipoarabinomannan testing for diagnosis of pulmonary tuberculosis in children: A prospective study. Lancet Glob. Health 2014, 2, e278-e284. [CrossRef]

27. Nicol, M.P.; Schumacher, S.G.; Workman, L.; Broger, T.; Baard, C.; Prins, M.; Bateman, L.; du Toit, E.; van Heerden, J.; Szekely, R.; et al. Accuracy of a novel urine test, Fujifilm SILVAMP tuberculosis lipoarabinomannan, for the diagnosis of pulmonary tuberculosis in children. Clin. Infect. Dis. 2020. [CrossRef]

28. Nkereuwem, E.; Togun, T.; Gomez, M.P.; Székely, R.; Macé, A.; Jobe, D.; Schumacher, S.G.; Kampmann, B.; Denkinger, C.M.; Abok, I.I.; et al. Comparing accuracy of lipoarabinomannan urine tests for diagnosis of pulmonary tuberculosis in children from four African countries: A cross-sectional study. Lancet Infect. Dis. 2020, 3099, 1-9. [CrossRef]

29. Paris, L.; Magni, R.; Zaidi, F.; Araujo, R.; Saini, N.; Harpole, M.; Coronel, J.; Kirwan, D.E.; Steinberg, H.; Gilman, R.H.; et al. Urine lipoarabinomannan glycan in HIV-negative patients with pulmonary tuberculosis correlates with disease severity. Sci. Transl. Med. 2017, 9. [CrossRef]

30. Jaganath, D.; Mupere, E. Childhood tuberculosis and malnutrition. J. Infect. Dis. 2012, 206, 1809-1815. [CrossRef]

31. Ibrahim, M.K.; Zambruni, M.; Melby, C.L.; Melby, P.C. Impact of childhood malnutrition on host defense and infection. Clin. Microbiol. Rev. 2017, 30, 919-971. [CrossRef] [PubMed]

32. Chandrasekaran, P.; Saravanan, N.; Bethunaickan, R.; Tripathy, S. Malnutrition: Modulator of immune responses in tuberculosis. Front. Immunol. 2017, 8, 1316. [CrossRef] [PubMed]

33. Broger, T.; Nicol, M.P.; Sigal, G.B.; Gotuzzo, E.; Zimmer, A.J.; Surtie, S.; Caceres-Nakiche, T.; Mantsoki, A.; Reipold, E.I.; Székely, R.; et al. Diagnostic accuracy of 3 urine lipoarabinomannan tuberculosis assays in HIV-negative outpatients. J. Clin. Investig. 2020, 130, 5756-5764. [CrossRef] [PubMed]

34. Bjerrum, S.; Broger, T.; Székely, R.; Mitarai, S.; Opintan, J.A.; Kenu, E.; Lartey, M.; Addo, K.K.; Chikamatsu, K.; Macé, A.; et al. Diagnostic accuracy of a novel and rapid lipoarabinomannan test for diagnosing tuberculosis among people with human immunodeficiency virus. Open Forum Infect. Dis. 2020, 7. [CrossRef] [PubMed]

35. World Health Organization. High-Priority Target Product Profiles for New Tuberculosis Diagnostics: Report of a Consensus Meeting; WHO: Geneva, Switzerland, 2014; pp. 1-96. 
36. Ricks, S.; Denkinger, C.M.; Schumacher, S.G.; Hallett, T.B.; Arinaminpathy, N. The potential impact of urine-LAM diagnostics on tuberculosis incidence and mortality: A modelling analysis. PLoS Med. 2020, 17, e1003466. [CrossRef]

37. Roya-Pabon, C.L.; Perez-Velez, C.M. Tuberculosis exposure, infection and disease in children: A systematic diagnostic approach. Pneumonia 2016, 8, 1-18. [CrossRef]

38. Broger, T.; Muyoyeta, M.; Kerkhoff, A.D.; Denkinger, C.M.; Moreau, E. Tuberculosis test results using fresh versus biobanked urine samples with FujiLAM. Lancet Infect. Dis. 2020, 20, 22-23. [CrossRef] 